\title{
Three Questions Concerning the Foundation of Multi-perspective Classification
}

\author{
Wan-Chen Lee \\ University of Washington iSchool \\ leew23@uw.edu
}

\begin{abstract}
This paper contributes to the discussion of the purposes and functions of multi-perspective classification by raising foundational questions. Starting from the motivations of developing multi-perspective classification, the author discusses questions concerning the accommodation of different perspectives. The discussion leads to the question "what is multi-perspective classification?" and envisions a possible amelioration. Through discussing the three questions concerning the foundation of multi-perspective classification, the goal is to identify challenges and thoughts for developing multi-perspective classification before focusing on specific implementations.
\end{abstract}

\section{Keywords}

Classification, multi-perspective.

\section{INTRODUCTION}

This paper contributes to the discussion of the purposes and functions of multi-perspective classification by raising foundational questions. Starting from the motivations of developing multi-perspective classification, the author looks at multi-perspective classification in different contexts, and explores the question of "why would we want, or not want multi-perspective classification?" Using three classification schemes and the LCSH as examples, the author discusses questions concerning the accommodation of different perspectives, such as the criteria for inclusion and exclusion of perspectives. The discussion leads to the question "what is multi-perspective classification?" and envisions a possible amelioration. The purpose of this paper is to discuss the foundation of multi-perspective classification through general but critical questions. The goal is to identify challenges and thoughts for developing multi-perspective classification before focusing on specific implementations.

Advances in Classification Research, 2017, October 27, 2017, Washington, DC, USA.

\section{THE MOTIVATION OF DEVELOPING MULTI- PERSPECTIVE CLASSIFICATION}

Previous studies (Mai, 2004; 2009; Miksa, 1998) review shifts of stances in classification theory: from the universe of knowledge stance to the pluralistic understanding stance, and beyond. As the foundation of early bibliographic classifications, the universe of knowledge stance seeks one neutral description of the accurate and objective reality for universal users. It was replaced by pluralistic understanding, which acknowledges that classifications, concepts, categories, meanings, and subjects are determined by the historical, social, and cultural context of user communities. The development of the Nippon Decimal Classification (NDC) and the New Classification Scheme for Chinese Libraries (CCL) are examples supporting this stance. The editors of the first edition of both schemes justified the necessity of developing local schemes by arguing how the adoption of the Dewey Decimal Classification (DDC) would fail to satisfy local needs (Mori, 1929; Liu, 1929). Feinberg (2007) suggests taking one step further from this pluralistic understanding. Instead of passively viewing value judgments as inevitable, she argues that classification can be arguments for interpretations. Classification frames the context of which users and information interact. By making the underlying perspective explicit, classificationists could make classifications active change agents for interpretations and work practices.

The desire for a multi-perspective classification arises from this aforementioned arc in classification theory evolution. In addition, multi-perspective classification can be a realization of cultural warrant. Beghtol (1986) emphasizes semantic warrant in classification, saying, "the personal and professional cultures of information seekers and information workers warrant the establishment of appropriate fields, terms, categories, or classes in a knowledge representation and organization system." (Beghtol, 2005, p.904). To accommodate different cultures in a classification, building a multi-cultural classification, which is a type of multiperspective classification, seems promising. However, culture is a complex concept with various dimensions. There is no consensus on the definition of culture in knowledge organization (Lee, 2015); and multiple cultures (e.g., discipline, nationality, ethnicity) may coexist within individuals. It remains unclear as of whose culture(s) and which dimensions of culture should be incorporated in 
classificatory decisions. While there are motivations supporting the development of multi-perspective classification, the approaches and challenges are pending for examination and discussion.

\section{MULTI-PERSPECTIVE CLASSIFICATION IN DIFFERENT CONTEXTS}

For places where the local perspective is the dominant perspective, a multi-perspective classification may have its backbone structure base on the local perspective; and include some instances of other perspectives to increase the diversity of the classification. For example, adding a list of Chinese Classics in their traditional order. In this case, the shift from a single-perspective classification to a multi-perspective classification would not cause significant changes. The local/dominant perspective remains prominent. For places where local perspective differs from the dominant perspective, the development of multi-perspective classification surfaces different concerns. Our example here is the CCL. To remain updated, a classification should be able to accommodate new knowledge from the local perspective while including the dominant perspective. A local community could adapt a classification built on the dominant perspective to satisfy local needs, or make changes to a classification built on local perspective to incorporate imported knowledge and perspectives. The influences, costs, motivations, and considerations concerning the development of multi-perspective classification may vary in different contexts.

\section{ACCOMODATING DIFFERENT PERSPECTIVES}

In a multi-perspective classification, how do we accommodate perspectives that prefer different class organizations? One example is the set of classes for "Chinese classics" in the CCL, the NDC, and the DDC. Chinese classics refer to thirteen seminal works. The titles span across multiple subjects, including philosophy, history, literature, rituals, and music. There are two sanctioned classifications for Chinese classics in the 2007 edition of CCL. Libraries can either (1) classify Chinese classics by subject (e.g., classify The Book of History under 621.11 History and geography -- Chinese history by period -Ancient (to 203 B.C.) -- The Book of History), or (2) collocate Chinese classics under 090 Generalities -Collected Chinese classics (e.g., 092: The Book of History). The 2014 edition of NDC collocates Chinese classics under class 123 Philosophy -- Oriental thought -- Chinese classics. The Book of History is under 123.2. The 23rd edition of DDC does not have a class for Chinese classics. If we look up the class numbers for the titles about one or multiple Chinese classics , we can identify popular classes, including 181.1 and 181.112 Philosophy \& psychology -- Ancient, medieval \& Eastern philosophy -- Eastern philosophy -- Far East and South Asia -- China and Korea -- Confucian philosophy; 299.512 and 299.51282 Religion -- Other religions -Religions not provided for elsewhere -- Religions of East and Southeast Asian origin -- Religions of Chinese origin -Confucianism -- sacred books, ...; 895.1 and 895.18
Literature -- Other literatures -- Literatures of East and Southeast Asia -- Chinese literature -- Chinese prose literature.

This example emphasizes the challenges of accommodating different perspectives in a classification. The CCL shows that multi-perspective does not necessarily mean multicultural. There can be different perspectives and preferred classifications within the same culture. Looking at the four perspectives presented in the three schemes, the NDC and one perspective in the CCL prefer collocating the Chinese classics. The former places Chinese classics under Philosophy; and the latter under Generalities. The DDC and the other perspective in the CCL classify Chinese classics by subject. In the CCL, Chinese classics are dispersed in a variety of classes: Philosophy, Social sciences, History and geography, Linguistics \& Literature, and Arts. In the DDC, most of the Chinese classics are under Literature, Philosophy \& psychology, and Religion. Further, only selective aspects of Chinese classics are presented.

\section{IMPORTED PERSPECTIVES}

To develop a multi-perspective classification, it is necessary to clarify the scope, and set the criteria for the inclusion and exclusion of perspectives. For situations of which a perspective is not well represented in local literature, classificationists should consider the pros and cons of importing such perspective. There is no guarantee of whether an imported perspective would thrive or go stale. A controlled vocabulary example is the LCSH "Comics, strips, etc." and its variant "Manga (Comics, strips, etc.)." While some perspectives differentiate comics from manga (Cohn, 2011), Manga is not a sanctioned heading in LCSH. The line between including and excluding a term representing another perspective could be subtle and debatable. Establishing the criteria for imported perspectives helps us explore and identify the prioritization of purposes for pursuing multi-perspective classification.

\section{DISCUSSION: WHAT IS MULTI-PERSPECTIVE CLASSIFICATION?}

In the previous sections, we see the motivations of including multiple perspectives in a classification; and the potential tension between the need for a classification to incorporate knowledge from other perspectives and the need to cater to local perspectives. The classifications of Chinese classics in three schemes highlight challenges of accommodating multiple perspectives in a classification. We can explore the criteria for the inclusion and exclusion of perspectives in a multi-perspective classification through cases like the manga instance above. These discussions lead to a fundamental question: what is multi-perspective classification? If the purpose of multi-perspective classification is to present voices from different perspectives instead of promoting one voice and silencing others, does the proportion of perspectives matter? To qualify as a perspective in a multiperspective classification, to what extent should a perspective be presented? Is a classification with one dominant perspective and limited instances of other 
perspectives considered a multi-perspective classification? Further, how do we technically accommodate multiple perspectives in one classification? If we consider classification as having mutually exclusive classes and assigning only one place for one thing, libraries should apply only one arrangement for each class, despite the various possible conceptual arrangements. If we include different perspectives, so one thing may be classified under multiple classes, is the end product a multi-perspective "classification?"

Given the above exploration, perhaps a possible form of multi-perspective classification is a classification providing limited sanctioned options for the organization the individual classes. The options present different perspectives and characteristics of division. Chinese classics in the CCL is an example of two sanctioned options for a class. This approach is a compromise between two extremes: the oneperspective-for-all classification, and classification incorporating perspectives without specific principles. On one hand, libraries must choose only one of the sanctioned options, so the classes are mutually exclusive, and the variations in practice are limited. Furthermore, if the options are provided by classificationists who design and maintain the classification, the classificatory rationale of different options would more likely be consistent and compatible with other parts of the classification. On the other hand, libraries are not limited by one perspective for all classes, and have the flexibility to choose the best option for individual classes to serve local needs. A classification may incorporate a combination of perspectives through the choices of sanctioned options for classes. It is clear from this brief exploration that there are, indeed, multiple perspectives on multi-perspective classification.

\section{REFERENCES}

Beghtol, C. (1986). Semantic validity: concepts of warrant in bibliographic classification systems. Library Resources and Technical Services 30(2), 109-125.

Beghtol, C. (2005). Ethical decision-making for knowledge representation and organization systems for global use. Journal of the American Society for Information Science and Technology 56(9), 903-912. https://doi.org/10.1002/asi.20184

Cohn, N. (2011). A different kind of cultural frame: An analysis of panels in American comics and Japanese manga. Image \& Narrative 12(1), 120-134.

Feinberg, M. (2007). Beyond retrieval: A proposal to expand the design space of classification. Proceedings of the North American Symposium on Knowledge Organization 1, (pp. 31-43.) https://doi.org/10.7152/nasko.v1i1.12832

Lee, W.-C. (2015). Culture and Classification: An Introduction to Thinking about Ethical Issues of Adopting Global Classification Standards to Local Environments. Knowledge Organization 42(5), 302-307.

Liu, K.-C. (1929). Zhongguo tu shu fen lei fa = A System of Book Classification for Chinese Libraries. Nanking: Jinling da xue tu shu guan.

Mai, J.-E. (2004). Classification in Context: Relativity, Reality, and Representation. Bibliographical Essay 31(1), 39-48.

Mai, J.-E. (2009). The Boundaries of Classification. Advances in Classification Research Online 20(1). https://doi.org/10.7152/acro.v20i1.12887

Miksa, F. L. (1998). The DDC, the universe of knowledge, and the post-modern library. Albany, N.Y.: Forest Press.

Mori, K. (1929). Nihon jisshin bunruiho: Wakan yosho kyoyo bunruihyo oyobi sakuin [Nippon Decimal Classification scheme: Classification table and index for Japanese, Chinese, and foreign books]. Osaka: Mamiya Shoten. 